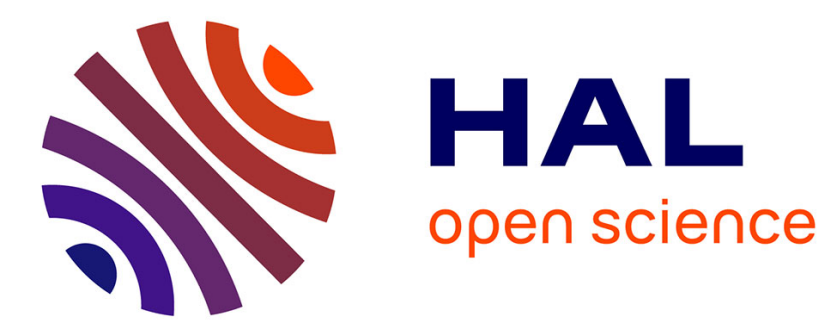

\title{
Les rebelles syriens d'Ahrar al-Sham : ressorts contextuels et organisationnels d'une déradicalisation en temps de guerre civile
}

\author{
Ahmad Abazeid, Thomas Pierret
}

\section{- To cite this version:}

Ahmad Abazeid, Thomas Pierret. Les rebelles syriens d'Ahrar al-Sham : ressorts contextuels et organisationnels d'une déradicalisation en temps de guerre civile. Critique Internationale, 2018, 78 (1), pp.63-84. 10.3917/crii.078.0063 . hal-01753400

\author{
HAL Id: hal-01753400 \\ https://hal.science/hal-01753400
}

Submitted on 29 Mar 2018

HAL is a multi-disciplinary open access archive for the deposit and dissemination of scientific research documents, whether they are published or not. The documents may come from teaching and research institutions in France or abroad, or from public or private research centers.
L'archive ouverte pluridisciplinaire HAL, est destinée au dépôt et à la diffusion de documents scientifiques de niveau recherche, publiés ou non, émanant des établissements d'enseignement et de recherche français ou étrangers, des laboratoires publics ou privés. 
Les rebelles syriens d'Ahrar al-Sham entre légitimités jihadiste et révolutionnaire

\begin{abstract}
NB : Ce texte est la version initiale (pré-évaluation), soumise le 3 décembre 2016, de l'article publié en mars 2018 dans Critique internationale $n^{\circ} 78$ sous le titre « Les rebelles syriens d'Ahrar al-Sham : ressorts contextuels et organisationnels d'une déradicalisation en temps de guerre civile »
\end{abstract}

\begin{abstract}
Ahmad Abazeid : Chercheur et essayiste indépendant, spécialiste de la révolution syrienne et des groupes insurgés. Il est l'auteur d'études publiées par Chatham House, Atlantic Council, Al Jazeera Centre for Studies, Omran Center for Strategic Studies et Toran for Strategic Studies.
\end{abstract}

Thomas Pierret : Chargé de recherche, Aix Marseille Université, CNRS, IREMAM, Aix-enProvence, France

Avec une force combattante estimée aux alentours de 20.000 hommes en 2016, le mouvement salafiste Ahrar al-Sham est, après l'État Islamique, la principale unité rebelle opérant en Syrie. Avec Fath al-Sham (ex-Front al-Nusra), Ahrar al-Sham est aussi la seule faction rebelle d'envergure réellement nationale, même si principalement ancrée dans le nord-ouest (Idlib, Hama et Alep). Toutefois, la raison spécifique pour laquelle nous nous penchons ici sur ce groupe est qu'il constitue un point d'observation privilégié des contradictions idéologiques qui traversent l'opposition armée syrienne. En effet, Ahrar al-Sham se situe à l'intersection des deux principales catégories de rebelles opérant dans le pays : les révolutionnaires et les jihadistes. ${ }^{1}$ Les premiers, même s'ils font éventuellement usage d'un lexique islamiste, définissent le domaine de la lutte en référence à la révolution syrienne de 2011 et prétendent par conséquent à une légitimité politique conférée par le peuple en révolte. Les seconds s'inscrivent au contraire dans un jihad transnational dont le conflit syrien n'est qu'une étape, et situent l'origine de la légitimité politique dans une avant-garde (eux-mêmes) qui a démontré son dévouement à la cause en prenant les armes bien avant le Printemps Arabe. Là où la posture des premiers induit la reconnaissance du principe de régime représentatif, celle des seconds mène à l'exclusivisme assumé qui caractérise notamment le système politique mis en place par l'État Islamique en Syrie et en Irak.

Le processus qui a conduit Ahrar al-Sham, issu du jihadisme, à embrasser une partie des idéaux « révolutionnaires » pour produire une synthèse « révisionniste ${ }^{2}$ apporte un éclairage nouveau sur le phénomène de «déradicalisation » ou de «modération » des mouvements islamistes. Avec Omar Ashour, nous définissons ici la déradicalisation comme «l'évolution vers l'acceptation de changements sociaux, politique et économiques graduels dans un contexte pluraliste $»^{3}$. Alors que s'agissant des islamistes, ce phénomène a principalement été abordé jusqu'à présent dans des contextes de consolidation autoritaire (ouverture du jeu

\footnotetext{
${ }^{1}$ International Crisis Group, "New approach in Southern Syria », Middle East Report ${ }^{\circ} 163$, 2 septembre 2015, https://d2071andvip0wj.cloudfront.net/163-new-approach-in-southernsyria.pdf.

${ }^{2}$ Sam Heller, « Ahrar al-Sham's revisionist Jihadism », War on the Rocks, 30 septembre 2015, http://warontherocks.com/2015/09/ahrar-al-shams-revisionist-jihadism/.

${ }^{3}$ Omar Ashour, « Deradicalization revisited », Monkey Cage, 18 février 2015, http://www.washingtonpost.com/blogs/monkey-cage/wp/2015/02/18/deradi- calization-revisited/.
} 
électoral ou défaite de l'opposition armée $)^{4}$ ou de transition démocratique ${ }^{5}$, Ahrar al-Sham constitue un cas, contre-intuitif, de déradicalisation en contexte de guerre civile, contexte où le sens commun pourrait suggérer que l'escalade de la violence se reflète nécessairement dans l'évolution idéologique des protagonistes.

Notre préoccupation centrale n'est pas ici d'étudier les origines du révisionnisme idéologique d'Ahrar al-Sham, ${ }^{6}$ d'examiner les détails de son contenu ${ }^{7}$ ou, encore moins, de déterminer s'il satisfait de quelconques critères de respectabilité du point de vue des acteurs occidentaux. ${ }^{8}$ Adoptant une perspective processuelle, nous nous intéressons plutôt aux luttes incessantes qui se déploient autour de cet exercice de redéfinition idéologique, des luttes qui impliquent factions rivales au sein du mouvement, groupes jihadistes et révolutionnaires extérieurs à ce dernier, et États étrangers. Nous montrerons que la posture idéologique d'Ahrar al-Sham est déterminée par la quête permanente d'une double légitimité, auprès des jihadistes et des révolutionnaires, mais qu'une combinaison de caractéristiques génétiques du groupe (partenariat avec des États régionaux, nature collégiale du leadership) et de développements contingents (réaction populaire anti-jihadiste, rivalités sur le terrain avec le Front al-Nusra) ont tendanciellement éloigné Ahrar al-Sham de la sphère jihadiste.

Après une première section consacrée à la cristallisation du révisionnisme d'Ahrar al-Sham entre 2012 et 2014, nous nous concentrerons sur la période ultérieure à travers deux sections thématiques. La seconde section se concentre sur les divisions internes au mouvement, partant du principe que les caractéristiques organisationnelles de la direction d'Ahrar al-Sham, à savoir sa forte institutionnalisation et sa collégialité, font que son évolution idéologique ne relève pas d'une simple adaptation stratégique au contexte mais procède aussi de dynamiques endogènes. La troisième section envisage pour sa part le problème sous l'angle des relations entre Ahrar al-Sham et ses partenaires extérieurs, jihadistes et révolutionnaires, au sein de la rébellion.

\section{La cristallisation d'une posture révisionniste (2012-2014)}

Les Bataillons Ahrar al-Sham sont créés fin 2011 par des militants jihadistes dont beaucoup ont été libérés de la prison de Seydnaya, près de Damas, dans les premiers mois du soulèvement. Vétérans du conflit irakien et, pour les plus anciens, de la précédente insurrection syrienne (1979-1982) et du premier jihad afghan, les cadres du groupe sont pour la plupart des ruraux diplômés. D'un point de vue idéologique, Ahrar al-Sham opte d'emblée

\footnotetext{
${ }^{4}$ Omar Ashour, The De-Radicalization of Jihadists. Transforming Armed Islamist Movements, Oxon, Routledge, 2009; Jilian Schwedler, "Can Islamists become moderates? Rethinking the inclusion-moderation hypothesis", World Politics, vol. 63, $n^{\circ} 2$, pp. 347-376.

${ }^{5}$ Jérôme Drevon, "The Emergence of Ex-Jihadi Political Parties in Post-Mubarak Egypt", Middle East Journal, vol. 69, n4, pp.511-526.

${ }^{6}$ Thomas Pierret, "Crise et déradicalisation : les rebelles syriens d'Ahrar al-Sham », Confluences Méditerranée, n94, 2015, pp.43-49.

${ }^{7}$ Heller, « Ahrar al-Sham's revisionist Jihadism ».

${ }^{8}$ Pour des perspectives opposées sur ce point, voir Charles Lister, "Syrian Islamists reach out to the US, but serious issues remain ", Brookings, 14 juillet 2015, https://www.brookings.edu/blog/markaz/2015/07/14/syrian-islamists-reach-out-to-the-u-sbut-serious-issues-remain/; Guido Steinberg, "Ahrar al-Sham: The 'Syrian Taliban' ", SWP

Comments, mai 2016, https://www.swpberlin.org/fileadmin/contents/products/comments/2016C27 sbg.pdf.
} 
pour un positionnement idiosyncratique au sein de l'insurrection syrienne, qui le place à midistance entre les factions, alors très majoritaires, adoptant le label d' " Armée Syrienne Libre » (ASL), et le Front al-Nusra, qui émerge au même moment en tant que franchise officieuse de l'État Islamique d'Irak, dont il se séparera l'année suivante. Optant pour une symbolique strictement islamique, Ahrar al-Sham n'adopte ni le label ASL, ni le drapeau national syrien dans sa version révolutionnaire, c'est-à-dire celui de l'ère pré-baasiste. Qui plus est, là où les factions ASL se présentent comme le simple bras armé de la révolution, Ahrar al-Sham se définit comme une faction idéologique dotée d'un projet politique (islamiste) propre, une prétention que manifeste, fin 2012, le remplacement du titre «bataillons » (kata'ib) par celui de «mouvement» (haraka). Toutefois, le groupe affecte aussi de se distancer du jihadisme transnational en insistant sur le cadre exclusivement syrien de ses opérations et son indépendance vis-à-vis d'al-Qaeda. En outre, durant sa première année d'existence, Ahrar al-Sham affiche un pragmatisme guère en phase avec l'orthodoxie jihadiste, se joignant à des membres du Conseil National Syrien (ancêtre de la Coalition Nationale) au sein d'un éphémère Front des Révolutionnaires de Syrie ou présidant à la constitution d'un Front Islamique Syrien dont la charte est critiquée par des idéologues radicaux pour son caractère syro-centré et son manque de clarté sur des questions comme l'infériorité légale des non-musulmans. ${ }^{9}$

Le révisionnisme d'Ahrar al-Sham trouve son origine dans le contexte de l'établissement du groupe. Celui-ci voit le jour au milieu d'une insurrection populaire qui ébranle à la fois le régime et les convictions de certains jihadistes. Déjà en proie aux doutes depuis l'échec du jihad irakien, ${ }^{10}$ qu'ils attribuent à l'exclusivisme de l'État Islamique d'Irak vis-à-vis des sunnites locaux, ces « révisionnistes » interrogent le modèle élitiste en vertu duquel seule l'avant-garde jihadiste serait un acteur de changement politique. Cette reconnaissance des vertus du peuple en armes explique vraisemblablement pourquoi les fondateurs adoptent une structure organisationnelle tout-à-fait singulière puisqu'elle combine l'avant-gardisme typique des factions jihadistes avec l'ancrage populaire des factions ASL. Ainsi, la hiérarchie supérieure du groupe est constituée du réseau de vétérans islamistes évoqué plus haut mais, au lieu de recruter individuellement des combattants et de les soumettre à un rigoureux processus de formation idéologique, comme le fait par exemple al-Nusra à l'époque, cette hiérarchie mobilise des bataillons locaux (ultérieurement regroupés en brigades) issus de la levée en masse observée à travers les campagnes du pays et dont les hommes de rang n'ont souvent qu'un rapport très distant, s'il existe, à l'idéologie jihadiste. Cette genèse marquera jusqu'à ce jour la structure du mouvement, caractérisée par une relative décentralisation et une direction collégiale représentée par un Conseil Consultatif (majlis al-shura) où siègent les représentants des principales brigades. Ces « grands électeurs » désignent un commandant en chef pour un mandat de douze mois, sans obligation de renouvellement puisque deux des quatre commandants en chef qu'a connu le mouvement à ce jour ont été remplacés à l'issue de leur premier mandat. Ce système contraste avec la structure de pouvoir ultra-personnalisée qui caractérise la plupart des autres factions rebelles.

Outre une stratégie de mobilisation qui, d'un point de vue jihadiste puriste, revient à préférer la quantité à la qualité, les fondateurs d'Ahrar al-Sham se distinguent de leur mouvance d'origine en faisant d'emblée le choix d'un partenariat étroit avec des États régionaux, en

\footnotetext{
${ }^{9}$ Aron Lund, "Syria's Salafi insurgents: the rise of the Syrian Islamic Front", UI Occasional Papers \#17, mars 2013, http://www.ui.se/eng/upl/files/86861.pdf.

${ }^{10}$ Entretien avec le directeur des relations extérieures d'Ahrar al-Sham Labib al-Nahhas, Istanbul, août 2015.
} 
l'occurrence la Turquie et le Qatar, ${ }^{11}$ dont le patronage encouragera plus avant les orientations réformistes du groupe. Le processus de déradicalisation de ce dernier ne s'opère toutefois pas de manière linéaire. Dès 2013, en effet, Ahrar al-Sham adopte une ligne plus dure dans un contexte de surenchère idéologique généralisée résultant de l'intervention du Hezbollah libanais aux côtés du régime, de l'offensive menée par l'Arabie Saoudite contre l'influence du Qatar et de ses alliés islamistes à travers la région, de l'annulation des frappes occidentales prévues contre le régime après le massacre à l'arme chimique du mois d'août, et de la montée en puissance de l'État Islamique d'Irak et du Levant (EIIL). C'est dans la foulée de ces développements que l'on voit Ahrar al-Sham prendre la tête d'initiatives comme le Communiqué $\mathrm{n}^{\circ} 1$, par lequel des factions rebelles dénient toute légitimité à la Coalition Nationale (récemment passée du giron de Doha à celui de Riyad) et rappellent l'impératif d'application de la charia, ${ }^{12}$ ou le Front Islamique, coalition des principales factions islamistes dont le programme, intitulé Projet de l'Umma, ne s'embarrasse guère de nuances dans son traitement de questions comme les combattants étrangers, dont la présence est saluée, où les notions de démocratie et d'État civil, rejetées sans ambages. ${ }^{13}$

On l'a dit, cette surenchère idéologique est en partie une réponse au défi constitué par les ambitions hégémoniques de l'EIIL, qu'une guerre ouverte finit par opposer aux autres factions rebelles à partir du début 2014. C'est pourtant durant ce même affrontement que s'affirment au sein d'Ahrar al-Sham de nouvelles révisions idéologiques s'opérant, cette fois, à rebours du maximalisme jihadiste. On voit ainsi le groupe signer, en mai, une Charte d'Honneur Révolutionnaire réaffirmant le caractère strictement syrien de la lutte dans un langage largement exempt de terminologie islamiste. ${ }^{14}$ Durant l'été, le mouvement apporte son soutien à l'éphémère initiative Wa 'tasimu (« Agrippez-vous »), qui vise à rassembler factions islamistes et non-islamistes derrière une direction politique commune. ${ }^{15} \mathrm{Ces}$ ouvertures sur le mainstream révolutionnaires sont vertement critiquées par les idéologues du Front al-Nusra, par ailleurs allié militaire d'Ahrar al-Sham contre le régime et l'EIIL, devenu en juin 2014 l'État Islamique (EI). ${ }^{16}$

Le revirement idéologique d'Ahrar al-Sham n'est pas sans lien avec des considérations prosaïques. Le groupe fait alors face à une dramatique diminution de ses ressources financières en raison de la capture par l'EI des puits de pétrole que le groupe contrôlait dans l'Est du pays, de sanctions décidées par le département américain du Trésor contre des financiers privés koweïtiens du groupe et d'une réduction du soutien qatari induite, elle aussi,

\footnotetext{
${ }^{11}$ Lund, "Syria's Salafi insurgents", p. 25.

${ }^{12}$ Aron Lund, "Islamist groups declare opposition to National Coalition and US strategy", Syria Comment, 24 septembre 2013, http://www.joshualandis.com/blog/major-rebelfactions-drop-exiles-go-full-islamist/.

${ }^{13}$ Aaron Zelin, "Rebels consolidating strength in Syria: the Islamic Front", Washington Institute, 3 décembre 2013, http://www.washingtoninstitute.org/policyanalysis/view/rebels-consolidating-strength-in-syria-the-islamic-front.

${ }^{14}$ Charles Lister, The Syrian Jihad: Al-Qaeda, the Islamic State and the Evolution of an Insurgency, London, Hurst, 2016, pp. 225-226.

${ }^{15}$ Idem, pp. 246-247.

${ }^{16}$ Idem, pp. 226-227 ; Sam Heller, « Ahrar al-Sham's Abu Yazan: It's our country and our revolution", 5 septembre 2014, https://abujamajem.wordpress.com/2014/09/05/ahrar-alshams-abu-yazan-its-our-country-and-our-revolution/.
} 
par des pressions américaines. ${ }^{17}$ Le groupe, qui ont le sait, s'était placé d'emblée dans une relation de coopération (et donc de dépendance) avec des États régionaux, doit à tout prix éviter d'être désigné comme une organisation terroriste par les USA pour préserver cette relation. Cependant, le changement de cap d'Ahrar al-Sham n'est pas une simple opération de relations publiques mais reflète une réelle évolution idéologique vis-à-vis des préceptes jihadistes.

Chez les leaders d'Ahrar al-Sham, la mise en cause du purisme jihadiste s'articule dans un débat, mené sur Twitter, avec les détracteurs radicaux du groupe. Le pragmatisme d'Ahrar alSham, justifie son chef Hassan 'Abbud (Abu Abdallah al-Hamawi), résulte du fait que le groupe mène le «jihad des gens ordinaires » et doit à ce titre s'adapter aux spécificités de sa société, là où ses détracteurs ne peuvent cultiver le purisme que parce qu'ils se sont coupés des masses pour conduire le «jihad des élites ». ${ }^{18}$ Muhammad al-Shami (Abu Yazan), qui émerge alors comme le principal idéologue du groupe, justifie quant à lui le rapprochement politique avec les factions non-islamistes en opposant un nécessaire « rassemblement sunnite » (al-istifaf al-sunni) par-delà les obédiences doctrinales (salafiste, soufie) au concept élitiste de «fraternité en méthode (salafiste-jihadiste) » (ukhuwwat al-manhaj). ${ }^{19}$ Outre le fait qu'il dresse une barrière infranchissable entre les jihadistes et les autres musulmans, ce concept serait, selon al-Shami, à l'origine du long aveuglement des jihadistes quant à la nature déviante de l'EI. «Méthodologistes » (manahija) devient ainsi une insulte désignant l'aile la plus radicale de l'insurrection. Plus remarquable encore est la répudiation par al-Shami de l'idée même de salafisme-jihadisme du fait, encore une fois, de sa nature élitiste :

Oui, j'étais un salafiste-jihadiste et je fus emprisonné pour cela par le régime. Aujourd'hui je demande pardon à Dieu et je me repens. Je te demande pardon, ô mon peuple, de t'avoir entraîné dans des batailles donquichottesques que nous aurions dû t'épargner. Je te demande pardon pour avoir un jour cherché à nous distinguer de toi. ${ }^{20}$

Une telle déclaration ne fait sans doute pas l'unanimité au sein d'Ahrar al-Sham, mais quelques jours après sa mise en ligne, un événement dramatique lui confère un statut tout particulier : le 9 septembre 2014, al-Shami et une dizaine de hauts-responsables d'Ahrar alSham, dont le commandant en chef 'Abbud et le chef religieux du mouvement Mahmud Tayba (Abu 'Abd al-Malik), périssent dans une mystérieuse explosion survenue lors d'une réunion. Les «commandants martyrs » seront dès lors littéralement iconisés sur les réseaux sociaux, et leur critique de l'exclusivisme jihadiste fournira, on le verra plus loin, de précieuses munitions aux partisans d'une lignée modérée au sein du mouvement.

Al-Shami laisse un mouvement dont l'armature conceptuelle est encore à l'état d'ébauche, son identité s'étant élaborée à travers un processus d'ajustement aux réalités pratiques du conflit syrien, plutôt qu'au départ d'une théorisation. La tentative la plus aboutie à ce jour de définir la spécificité praxéologique d'Ahrar al-Sham revient au chef des relations publiques Anas Najib. Celui-ci définit le mode d'action d'Ahrar al-Sham comme « concordiste» (tawfiqi), signifiant par-là qu'il se situe à mi-chemin entre celui des jihadistes et celui des « réformistes », c'est-à-dire des Frères Musulmans. Certaines des options détaillées par Najib ne sont qu'une traduction de la réalité du mouvement depuis 2012. C'est par exemple le cas

\footnotetext{
${ }^{17}$ Entretien avec un membre de la Coalition Nationale Syrienne, Paris, juillet 2014.

${ }^{18}$ Pierret, "Crise et déradicalisation", p. 46.

${ }^{19}$ Heller, "Ahrar al-Sham's Abu Yazan".

${ }^{20}$ Ibid.
} 
du principe de complémentarité entre le « jihad populaire » des réformistes et le «jihad élitiste » des jihadistes, qui reflète la combinaison d'un leadership avant-gardiste et de bataillons issus des communautés locales. De même, tandis que les réformistes rejettent la présence des combattants étrangers et que les jihadistes les mettent à l'avant-plan, Ahrar alSham les accepte tout en leur fermant l'accès aux échelons les plus élevés de sa hiérarchie. Dans l'hypothèse de l'établissement d'un système parlementaire, Najib affirme que son mouvement refuserait à la fois de se joindre aux réformistes dans leur engagement parlementaire, et de faire écho aux jihadistes lorsqu'ils excommunient ceux qui se livrent à cette activité. $^{21}$

Un tel positionnement est moins intéressant en ce qu'il permettrait d'entrevoir le comportement futur d'Ahrar al-Sham (l'établissement d'un réel système parlementaire en Syrie est pour l'heure totalement utopique) qu'en ce qu'il révèle les contradictions d'un groupe tiraillé entre les doctrines jihadistes dont il est issu et la volonté d'intégrer les revendications de représentation politique exprimées par le mouvement révolutionnaire. Résoudre ces contradictions est d'autant moins aisé, on va le voir, que le leadership d'Ahrar al-Sham est lui-même divisé sur la voie à emprunter.

\section{Un factionnalisme interne de nature idéologique}

À la différence des Frères Musulmans syriens, victimes depuis les années 1970 de plusieurs schismes fondés sur des identités régionales, la hiérarchie d'Ahrar al-Sham est le théâtre d'une rivalité de nature idéologique entre des ailes que l'on peut grosso modo qualifier de « dure » et de «modérée ». Le débat ne porte pas sur une remise en cause fondamentale de l'option révisionniste fondatrice mais concerne plutôt l'opportunité d'un approfondissement de ces révisions idéologiques pour éloigner le groupe de ses alliés jihadistes et le rapprocher en conséquence du mainstream révolutionnaire sur des thèmes tels que les symboles nationaux, la souveraineté populaire, et l'alliance avec les États régionaux.

Cette lutte pour la définition de l'identité d'Ahrar al-Sham éclate au grand jour après la décapitation du leadership en septembre 2014. À cette occasion, le mouvement donne la preuve de sa robustesse institutionnelle lorsque dès le lendemain de l'attentat, le Conseil Consultatif désigne un nouveau chef en la personne de Hachim al-Cheikh (Abu Jabir). Durant l'année qu'il passe à la tête du mouvement, al-Cheikh donne un coup de barre à droite en désignant des faucons notoires à la tête de l'aile militaire (Abu Salih al-Tahhan), du Bureau Chariatique (Abu Muhammad al-Sadiq) et de la brigade frontalière d'Idlib (Abu Khuzayma). Parallèlement, des commandants de brigade proches des révolutionnaires (Abu Mahmud Tel 'Ada, Abu al-Bara' Ma'rchimarin) sont limogés. En réaction, des figures historiques d'Ahrar al-Sham connues pour leur sensibilité révisionniste (Abu al-'Abbas al-Shami, Abu 'Abd alRahman al-Suri) quittent le mouvement pour fonder Jaysh al-Cham, une formation d'orientation plus distinctement islamo-nationaliste. ${ }^{22}$

Toujours durant le mandat d'al-Cheikh, des tensions croissantes apparaissent également entre les leaders des ailes militaire et religieuse du mouvement, d'une part, et les membres de son

\footnotetext{
${ }^{21}$ Page Facebook de Rushd (centre d'études et de formation proche d'Ahrar al-Sham), 28 février 2015, https://www.facebook.com/Rashad.center/ posts/782273521864514.

${ }^{22}$ Ahmad Abazeid, "Ahrar al-Sham après une longue année" (en arabe), Omran for Strategic Studies, 30 septembre 2015, pp.6-11, https://www.omrandirasat.org/الأبحاث/الدر اسات fitml.
} 
bureau politique, d'autre part, auquel les premiers reprochent notamment de chercher à soigner l'image du groupe auprès des décideurs occidentaux à travers des éditoriaux policés publiés dans le Washington Post et le Telegraph. ${ }^{23}$ Alors que les bureaux politiques des autres factions rebelles syriennes en sont souvent réduits à de simples fonctions de relations publiques, celui d'Ahrar al-Sham s'affirme progressivement comme capable de définir une ligne politique relativement autonome et, à ce titre, contestée par les doctrinaires. Pour ces derniers, l'assertivité croissante du bureau politique est la rançon de l'expansion du mouvement puisque la ligne réformiste y est principalement défendue par les frères Kinan et Labib al-Nahhas (Abu 'Azzam al-Ansari et Abu 'Izz al-Din), qui ont intégré Ahrar al-Sham fin 2014 lorsque la Brigade al-Haqq de Homs a fusionné avec le mouvement. ${ }^{24} \mathrm{Ce}$ cas n'est pas isolé : comme al-Haqq, la plupart des factions absorbées par Ahrar al-Sham depuis 2014 (dont en particulier Suqur al-Cham, qui prête allégeance en mars 2015) ne sont pas d'obédience jihadiste, pour la bonne et simple raison que les factions radicales désireuses de se fondre dans un poids lourd de l'insurrection n'ont que peu de sympathie pour le révisionnisme d'Ahrar al-Sham et préfèrent se tourner vers l'EI ou al-Nusra.

À la fin du mandat d'al-Cheikh en septembre 2015, le Conseil Consultatif lui choisit pour successeur Muhannad al-Masri (Abu Yahya al-Hamawi), ${ }^{25}$ qui préside, à l'inverse de son prédécesseur, à une ère d'affaiblissement des faucons. Que ces derniers aient perdu la main en dépit des douze mois durant lesquels ils avaient dominé l'appareil du mouvement s'explique par deux facteurs. Le premier est que s'il désigne les chefs des branches exécutives du mouvement, le commandant en chef n'est pas autorisé à modifier la composition du Conseil Consultatif, qui est son propre maître en la matière et dont le bloc modéré, toujours demeuré solide, s'est renforcé avec l'absorption de la Brigade al-Haqq et de Suqur al-Sham. Le deuxième facteur est l'évolution du contexte international et plus particulièrement de la posture du sponsor turc, lequel prépare alors une opération militaire dans le Nord de la Syrie, opération qui sera finalement reportée d'un an en raison de l'intervention russe et des hésitations américaines. Or, Ankara a pris ombrage des réticences de l'aile dure d'Ahrar alSham vis-à-vis de son projet, réticences qui se manifestent notamment à travers la fatwa d'un char ' $i$ égyptien déclarant illicite la coopération avec les troupes turques. ${ }^{26} \mathrm{C}$ 'est que, du point de vue des faucons du mouvement, si recevoir un soutien logistique des services secrets turcs est une chose, servir de supplétifs à une armée de l'OTAN contre d'autres islamistes sunnites, fussent-ils ennemis, en est une autre.

Après son élection, al-Masri agit avec prudence par souci de préserver la cohésion du mouvement mais s'attache néanmoins à affaiblir l'aile dure du groupe. Cette entreprise vise d'abord Abu Muhammad al-Sadiq et son Bureau Chariatique, qui est dissout et dont les prérogatives sont distribuées entre trois nouveaux organismes confiés à des modérés (Instance de Prédication et de Guidance dirigée par Abu Muwaffaq al-Sadiq Hama et Instance Judiciaire par Ahmad Najib) ou, du moins, reflétant des sensibilités diverses (le Conseil

\footnotetext{
${ }^{23}$ Ibid. ; Pierret, "Crise et déradicalisation", p. 43;

${ }^{24}$ Abazeid, "Ahrar al-Sham après une longue année", p.8.

${ }^{25}$ Aron Lund, "Abu Yahia al-Hamawi, Ahrar al-Sham's New Leader", Syria Comment, 12 septembre 2015, http://www.joshualandis.com/blog/abu-yahia-al-hamawi-ahrar-al-shamsnew-leader/.

${ }^{26}$ Abazeid, « Ahrar al-Sham après une longue année », p. 24.
} 
Chariatique de cinq membres où se côtoient notamment le radical 'Abd al-Razzaq al-Mahdi et Ayman Harush, marginalisé sous al-Cheikh). ${ }^{27}$

Plus délicat se révèle le limogeage, en juin 2016, du chef militaire Abu Salih al-Tahhan, alors qu'entretemps, les faucons se sont raidis face à la participation du mouvement à la conférence de Riyad (cfr infra). En réaction à la mise à l'écart d'al-Tahhan, des fidèles de ce dernier (Abu Khuzayma, Abu al-'Abbas Halab), répondent par l'arrestation de plusieurs colombes (Abu Anas Saraqib, Abu 'Abd al-Rahman al-Hamawi). Le conflit est rapidement désamorcé mais donne à al-Masri l'occasion d'avancer ses pions, encore une fois avec la pondération requise pour éviter d'approfondir la fracture : si al-Tahhan est réintégré au poste, moins influent, de vice-chef du mouvement aux affaires militaires, Abu Khuzayma se voit retirer le secteur frontalier au profit d'Abu al-Bara' Ma'rshimarin, un modéré dégradé sous le mandat d'alCheikh. ${ }^{28}$ Le même mois, Jaysh al-Sham, une faction dissidente dont on se souvient qu'elle avait été fondée par des leaders de l'aile modérée, annonce son retour dans le giron d'Ahrar al-Sham. $^{29}$

Les remaniements opérés par al-Masri ont offert au Bureau Politique du mouvement l'occasion d'affirmer un peu plus son rôle, une émancipation qui demeure controversée sur le plan interne et rencontre d'évidente limites. Ces dynamiques contradictoires sont illustrées par la participation d'Ahrar al-Sham à la conférence tenue par l'opposition à Riyad en décembre 2015 afin d'établir une Haute Instance de Négociation (HIN), délégation unifiée de l'opposition politique et militaire qui participera, à partir de janvier 2016, aux négociations de Genève 3. L'attitude d'Ahrar al-Sham à cette occasion est symptomatique du souci du mouvement de préserver sa double légitimité révolutionnaire et jihadiste, au prix d'une position souvent brumeuse.

En acceptant l'invitation qui lui est adressée par les Saoudiens, la faction rebelle fait initialement montre d'un pragmatisme considérable, et donc encore une fois dénoncé par alNusra, puisqu'elle consent au principe de rejoindre les autres composantes de l'opposition, dont des laïcs, au sein d'un organisme supposé négocier avec le régime une solution politique au conflit. Toutefois, Ahrar al-Sham se retire in extremis des discussions, arguant de la présence de pseudo-opposants inféodés au régime, de l'absence de référence à l'identité islamique du peuple syrien dans la déclaration finale, et de la faiblesse de la représentation accordées aux factions rebelles dans l'HIN. Une fois encore, l'épisode souligne non seulement les exigences contradictoires auxquelles fait face le mouvement mais aussi ses divisions internes, puisque le retrait est annoncé par la direction centrale au moment même où les membres du Bureau Politique, qui représentent le groupe à la conférence, donnent leur accord aux conclusions de cette dernière. ${ }^{30}$

Pour compliquer encore les choses, le refus d'Ahrar al-Sham de participer à l'HIN ne vaut pas clarification définitive de sa position concernant le processus politique. Ainsi, bien que ne prenant pas part aux discussions de Genève 3 , le groupe se déclare favorable à « tout

\footnotetext{
27 "Ahrar al-Sham dissout son Bureau Chariatique », Al-Durar al-Shamiyya, 18 novembre 2015, http://eldorar.com/node/91105.

${ }^{28}$ Entretien avec une source proche d'Ahrar al-Sham, Alep, août 2016.

${ }^{29}$ " Jaysh al-Sham se rallie à Ahrar al-Sham », Al-Durar al-Shamiyya, 19 juin 2016, http://eldorar.com/node/99611.

${ }^{30}$ "Ahrar se retire de la conférence de Riyad ", Zaman al-Wasl, 10 décembre 2015, https://www.zamanalwsl.net/news/66853.html.
} 
processus politique qui puisse réaliser les objectifs de la révolution syrienne », et exprime son soutien au HIN face aux pressions russo-américaines visant à lui imposer de revoir à la baisse la revendication de « chute du régime $» .{ }^{31}$ Lorsque les discussions entre Washington et Moscou aboutissent, fin février, à la proclamation d'une trêve sur l'ensemble des fronts, Ahrar al-Sham opte une nouvelle fois pour une position d'entre-deux, n'acceptant ni ne refusant officiellement la cessation des hostilités, tout en l'observant assez largement jusqu'au mois d'avril et en dénonçant ses violations par les loyalistes. ${ }^{32}$

Durant l'été 2016, enhardi par l'affaiblissement des faucons à l'intérieur du mouvement, le membre du bureau politique Labib al-Nahhas affirme encore davantage la ligne réformiste en se déclarant favorable à une forme de démocratie procédurale garantissant « liberté, État de droit, alternance (...), et possibilité pour les Syriens de choisir leurs dirigeants et représentants $» .{ }^{33}$ Favorable de longue date à l'adoption officielle du drapeau national, alNahhas insiste aussi qu'il «n'y a plus de raison acceptable de ne pas unifier la révolution sous son drapeau, qui sera, si Dieu le veut, le drapeau de la Syrie le jour où vaincra la révolution. ${ }^{34}$

Cherchant à peser sur les équilibres internes d'Ahrar al-Sham au profit des faucons, al-Nusra (tout juste rebaptisé Fath al-Sham) dénonce les positions d'al-Nahhas et pointe du doigt les contradictions qui continueraient d'opposer les branches politique et militaire du mouvement. ${ }^{35}$ Dans cet affrontement, al-Nahhas est vulnérable, puisqu'il est dépourvu de légitimité jihadiste antérieure à 2011 et n'est pas une figure historique d'Ahrar al-Sham. Toutefois, il reçoit le soutien de figures majeures de son groupe : al-Masri lui-même ${ }^{36}$, et surtout le conseiller du politburo Iyyad al-Sha'ar (Abu al-Hassan al-Tabuki), un vétéran du jihad afghan des années 1980 qui se fend d'un très explicite hashtag \#Labib_al-

Nahhas_me_représente. ${ }^{37}$ Les vivants ne sont pas les seuls à venir au secours d'al-Nahhas, qui prend soin de légitimer ses prises de positions en les présentant comme la continuation des révisions idéologiques des leaders martyrs assassinés en septembre $2014 .^{38}$

Une nouvelle étape dans l'affaiblissement des radicaux se déroule en parallèle et résulte, comme un an auparavant, du projet d'intervention turque. Dès le printemps 2016, des combattants d'Ahrar al-Sham avaient combattus l'État Islamique le long de la frontière aux

\footnotetext{
${ }^{31}$ « Ahrar al-Sham soutien le processus politique et refuse les pressions internationales contre le HIN ", Al-Durar al-Shamiyya, http://eldorar.com/node/94009.

${ }^{32}$ Aron Lund, "Truce tests relations between Islamist giants », Syria Deeply, 9 mars 2016.

${ }^{33}$ Al-Hayat, 10 août 2016, http://www.alhayat.com/m/story/16820038\#sthash.TYaS5U3Z.dpbs.

${ }^{34}$ Labib al-Nahhas, "Les leçons de la tentative manquée de coup d’État en Turquie pour la révolution et ses dirigeants » (en arabe), Al-Durar al-Shamiyya, 16 juillet 2016, http://eldorar.com/node/100530.

35 "Le porte-parole de Fath al-Sham dénonce la confusion régnant au sein d'Ahrar al-Sham et les contradictions entre ses ailes (politiques et militaires) " (en arabe), Al-Durar al-

Shamiyya, 12 août 2016, http://eldorar.com/node/101641.

${ }^{36}$ " Le chef d'Ahrar al-Sham clôt le débat sur les initiatives de sa branche politique " (en arabe), Al-Durar al-Shamiyya, 15 août 2016, http://eldorar.com/node/101768.

${ }^{37}$ "Le porte-parole de Fath al-Sham dénonce ... "

${ }^{38}$ Voir par exemple l'entretien accordé par al-Nahhas au magazine des Frères Musulmans syriens Al-'Ahd, 22 mars 2016, http://eldorar.com/node/96062.
} 
côtés de rebelles syriens affiliés au programme Train and Equip du Pentagone et bénéficiant occasionnellement du soutien de l'aviation américaine et de l'artillerie turque. Le 24 août, Ankara lance en territoire syrien l'opération Bouclier de l'Euphrate dans le but de chasser l'État islamique de la zone frontalière de Jrabulus et, en poussant plus au sud vers al-Bab, d'empêcher l'établissement d'une continuité territoriale entre les deux zones tenues par les nationalistes kurdes du PYD. Bien que l'acceptation par Ahrar al-Sham du principe d'une intervention turque soit de notoriété publique depuis un an, la question demeure de toute évidence épineuse au sein du mouvement. En témoignent la grande discrétion de ce dernier concernant l'appui donné par ses combattants à l'armée turque et le fait que son Conseil Chariatique mettra un mois à publier une fatwa déclarant licite la participation à Bouclier de l'Euphrate. ${ }^{39}$ Ce retard est le produit de tensions internes qu'illustreront la défection, en réaction à cette fatwa, du bataillon alépin Mujahidun Ashidda' et des char 'is égyptiens Abu Yaqdhan et Abu Shu'ayb al-Masri. ${ }^{40}$

Les durs n'ont toutefois pas dit leur dernier mot, et cela pour une raison identique à celle qui avait permis à leurs adversaires de revenir à l'avant-plan l'année précédente, à savoir la nature collégiale de la structure décisionnelle du groupe. Outre l'intervention turque, les débats internes s'avivent à propos d'une éventuelle fusion, soutenue par les durs et rejetées par les modérées, avec Fath al-Sham. Le mandat d'al-Masri arrivant à terme en septembre, les faucons proposent les candidatures d'al-Cheikh et d'al-Tahhan, tandis qu'après deux mois de blocages, leurs adversaires obtiennent l'élection d'Ali al-'Umar (Abu 'Ammar), le vicecommandant en chef du mouvement. Ce dernier est une figure relativement faible puisqu'il n'a rejoint Ahrar al-Sham qu'en mars 2015 lorsque son ancienne formation, Suqur al-Sham, a prêté allégeance au groupe. Les faucons ont donc des raisons de penser qu'al-'Umar sera moins un arbitre qu'un relais de l'influence de leurs rivaux, si bien que dix d'entre eux (dont al-Cheikh, al-Tahhan, al-Sadiq, et Abu Khuzayma) annoncent la suspension de leur participation au Conseil Consultatif. ${ }^{41}$ Deux ans après la décapitation d'Ahrar al-Sham, la rivalité entre ses deux factions rivales atteint donc une acuité sans précédent, et ce alors que la rébellion subit des revers dévastateurs à Alep.

Dans les pages précédentes, on a essentiellement présenté les jihadistes d'al-Nusra/Fath alSham comme une contrainte idéologique pour Ahrar al-Sham, du fait de leur rôle de censeurs vis-à-vis des initiatives pragmatiques du groupe et en particulier de son aile modérée.

Toutefois, les récentes discussions sur une fusion des deux mouvements rappellent que leurs relations relèvent aussi d'un rapport de force qui n'est pas seulement discursif mais se déploie également de manière concrète sur le terrain. Une dimension-clé du problème, qui fait l'objet de la prochaine section, est ici la question des relations avec ces tierces-parties que sont les autres factions rebelles.

\section{Entre jihadistes et Armée Libre}

\footnotetext{
39 "Le Conseil Chariatique d'Ahrar al-Sham légitime le combat aux côtés de l'armée turque » (en arabe), Al-Durar al-Shamiyya, http://eldorar.com/node/103206.

${ }^{40}$ Tweets des intéressés sur leurs comptes personnels, 21 septembre https://twitter.com/aboalyakzan/status/778682002266390528 et https://twitter.com/talha1331/status/778681836855627777.

${ }^{41}$ " Ahrar al-Sham : crise au Conseil Consultatif » (en arabe), Almodon, 29 novembre 2016, http://www.almodon.com/arabworld/2016/11/29/أحر ار-الثام-أزمة_مجلس-الثورى
} 
On se souvient que la décapitation d'Ahrar al-Sham en septembre 2014 survient dans un contexte de réaction anti-jihadiste issu de l'affrontement entre les rebelles et l'EI. Dans les mois qui suivent l'élection d'al-Cheikh, cette réaction populaire s'accentue encore face à la radicalisation, cette fois, du Front al-Nusra. En effet, aux victoires fulgurantes remportées par l'EI en Irak et en Syrie à la mi-2014, le chef d'al-Nusra Abu Muhammad al-Jolani répond en promettant à ses fidèles l'établissement en Syrie d'un «Émirat». Cette annonce, effectuée lors d'une réunion secrète mais révélée par la fuite d'un enregistrement sonore, est suivie d'une surenchère radicale se manifestant notamment par une campagne militaire qui, durant l'hiver 2014-2015, aboutit à la destruction de plusieurs factions ASL d'Idlib et Alep. Les principales victimes d'al-Nusra sont le Front des Révolutionnaires de Syrie (FRS) et le Mouvement Hazm, auxquels les jihadistes reprochent leur corruption et le soutien qu'ils reçoivent de la CIA via le Müşterek Operasyon Merkezi (MOM, «Centre des Opérations Conjointes »), la chambre d'opération des États sponsors de la rébellion basée en Turquie. ${ }^{42}$

La campagne menée par al-Nusra provoque des incidents localisés avec Ahrar al-Sham, dont certaines unités sont accusés par la branche syrienne d'al-Qaeda de protéger des fugitifs du FRS et de Hazm, en sus du fait que des factions autrefois alliées à ces derniers (Ahrar al-Jabal al-Wustani, Maghawir al-Islam) prêtent allégeance à Ahrar al-Sham pour se protéger d'alNusra. Par ailleurs, cette période voit aussi la formation jihadiste étendre son réseau de tribunaux chariatiques (dur al-qada, «Maisons de Justice ») dans les zones d'influence de l'organe judiciaire et exécutif d'Ahrar al-Sham dans la région, l'Instance Islamique pour la Gestion des Territoires Libérés, dont un juge est tué par des membres d'al-Nusra en février $2015 .^{43}$

En dépit de ces accrochages, l'attitude générale d'Ahrar al-Sham face aux menées d'al-Nusra relève de la neutralité négative, le mouvement se bornant à critiquer l'intransigeance des jihadistes et à annoncer des initiatives d'interposition guère suivies d'effet. C'est qu'Ahrar alSham cherche à tout prix à éviter la confrontation, du fait des réticences idéologiques d'une partie de ses membres mais aussi parce que, d'un point de vue militaire, le mouvement ne peut se passer du concours des jihadistes à l'heure où se prépare une offensive majeure contre le régime dans la province d'Idlib. En mars 2015, en effet, l'établissement de Jaysh al-Fath («Armée de la Conquête »), une coalition dont Ahrar al-Sham et le Front al-Nusra constituent les piliers, donne le signal d'une campagne qui conduira à la capture de la quasi-totalité des positions loyalistes dans la province, dont son chef-lieu.

Il faut y insister, le choix fait par Ahrar al-Sham de participer à une coalition exclusivement islamiste (pour des raisons idéologiques, al-Nusra s'est opposé à l'inclusion de factions soutenues par le MOM) reflète la réalité militaire de la province d'Idlib. Dans cette dernière en effet, l'ASL est trop faible (a fortiori depuis l'épuration menée par al-Nusra en 2014-2015) pour constituer un partenaire militaire crédible. À Alep, en revanche, ce sont des groupes modérés qui constituent Fath Halab, principale alliance rebelle de la province établie en avril 2015 pour y prolonger, sans succès, les victoires militaires enregistrées à Idlib. Refusant de signer la charte distinctement non-idéologique de Fath Halab, al-Nusra crée une chambre d'opération parallèle d'ampleur plus modeste, Ansar al-Charia. Fidèle à son ambivalence, Ahrar al-Sham décide tout simplement de rejoindre simultanément dans les deux coalitions. ${ }^{44}$

\footnotetext{
${ }^{42}$ Abazeid, « Ahrar al-Sham après une longue année », pp. 26-31.

${ }^{43}$ Ibid., pp. 36-37.

${ }^{44}$ Ibid., p. 15.
} 
S'il n'est pas question de remettre en cause l'alliance tactique avec al-Nusra, donc, Ahrar alSham estime néanmoins nécessaire de soigner sa légitimité révolutionnaire en faisant écho à un mouvement d'opinion qui, contre les excès jihadistes, réaffirme les symboles de la révolution de 2011. Ainsi, lors du quatrième anniversaire de cette dernière en mars 2015, des activistes lancent sur les réseaux sociaux la campagne \#lève_le_drapeau_de_ta_révolution, laquelle reçoit une réponse favorable de la part de plusieurs commandants rebelles, dont un d'Ahrar al-Sham agissant à titre individuel (Husam Salam alias Abu Bakr), prennent la pose avec ledit drapeau. ${ }^{45}$ La réaction anti-jihadiste qui s'affirme à l'époque n'est donc pas le seul fait de quelques idéalistes mais est notamment animée par une alliance objective de cadres et propagandistes issus d'autres traditions islamistes : élite religieuse traditionnelle, Frères Musulmans ou encore salafistes activistes d'obédience sururiste. ${ }^{46}$ Fait remarquable, ce « lobby » anti-jihadiste comprend aussi Huzayfa 'Azzam, fils de la figure fondatrice du jihadisme transnational Abdallah 'Azzam, qui décrit l'exclusivisme d'al-Nusra comme une déviation de l'approche promue par son père. ${ }^{47}$

Tel est l'environnement idéologique dans lequel Ahrar al-Sham choisit, en mars 2015, d'annoncer sa fusion avec Suqur al-Sham sous un nouveau slogan, «Révolution du Peuple », qui rompt clairement avec le cadre conceptuel jihadiste 48 : révolution », plutôt que «jihad», est le nom de la lutte, ipso facto consignée au territoire syrien et au renversement d'Assad ; le « peuple », non les « frères en méthode », sont les détenteurs de l'agentivité politique. Cette évolution lexicale par rapport à la connotation transnationale du Projet de l'Oumma de 2013 survient, il faut le souligner, sous le mandat d'al-Cheikh et donc de l'aile dure d'Ahrar alCham. C'est là un signe que le mouvement dans son ensemble, plutôt que sa seule aile réformiste, est capable d'ajustements pragmatiques aux transformations de son environnement idéologique.

Passées les victoires militaires de la campagne d'Idlib, des divergences se font jour entre Ahrar al-Sham et les jihadistes quant à l'administration de la ville. Bien que les premiers obtiennent le poste de gouverneur pour l'un de leurs membres eu égard à leur statut de premier contributeur aux effectifs de Jaysh al-Fath, ils choisissent, encore une fois par souci d'éviter la confrontation, de n'adresser qu'une opposition rhétorique aux dérives doctrinaires de leurs alliés. Ceux-ci mettent par exemple en place une police spécifiquement dédiée au contrôle des tenues féminines dans l'espace public et, lors du cinquième anniversaire de la révolution en mars 2016, répriment des manifestants arborant le drapeau national. Si le chef d'Ahrar al-Sham exprime son soutien aux manifestants (« le peuple manifestera en brandissant le drapeau de la révolution », déclare-t-il à Al Jazeera, « et personne ne pourra l'en empêcher »), ${ }^{49}$ son groupe se garde d'intervenir sur le terrain. ${ }^{50}$

\footnotetext{
${ }^{45}$ Entretien avec le commandant Husam Abu Bakr d'Ahrar al-Sham, 2 avril 2015, https://www.youtube.com/watch?v=2ZZDLz8SrcA.

${ }^{46}$ Voir notamment les tweets des cheikhs Hassan al-Dughaym, Hassan al-Jaja ou encore Mujahid Ma'mun Dayraniyya.

${ }^{47}$ Voir en particulier son long récit à charge de la « campagne de l'Émirat » menée par alNusra, diffusé sur Twitter en mars 2016.

${ }^{48}$ Abazeid, "Ahrar al-Sham après une longue année ", p.37-40.

${ }^{49}$ Muhannad al-Masri, entretien avec Al Jazeera, 16 mars 2016, https://www.youtube.com/watch?v=xEI076MGxic.

${ }^{50}$ Lund, «Truce tests relations ...»; entretien avec Bilal Jabiro, Alep, août 2016.
} 
Les factions jihadistes imposent également leur volonté concernant le refus d'autoriser l'entrée à Idlib des agences du Gouvernement Intérimaire lié à la Coalition Nationale Syrienne. ${ }^{51}$

À l'échelle de la province, les relations entre Ahrar al-Sham et le Front al-Nusra se tendent après la conquête d'Idlib en raison d'une donnée structurelle. En effet, la destruction des principales factions ASL de la région par les jihadistes durant l'hiver 2014-2015 laisse les deux piliers de Jaysh al-Fath dans une situation de concurrence directe. Ce contexte est propice à l'éclatement de nouveaux affrontements localisés entre les deux groupes (à Kafr Nubbul en juillet 2015, à Dana en septembre suivant, à Salqin en janvier 2016) où se mêlent ambitions d'al-Nusra d'étendre son appareil judiciaire aux dépens d'Ahrar al-Sham, accusations contre ce dernier d'abriter d'anciens éléments des factions « pro-américaines », et soutien d'Ahrar al-Sham aux civils protestants contre les exactions des jihadistes envers des jeunes filles jugées «indécentes ». ${ }^{52}$ À partir de la fin 2015, en outre, al-Nusra mène une nouvelle série d'attaques contre des factions soutenues par le MOM, attaques dont deux (contre la Brigade al-Wusta en décembre 2015, et Jaysh al-Tahrir en juillet 2016) suscitent cette fois un réel effort d'interposition armée de la part d'Ahrar al-Sham. ${ }^{53}$ Deux facteurs entrent en compte dans le durcissement de l'attitude ce dernier. Premièrement, l'agressivité d'al-Nusra envers les autres factions rebelles et son refus de s'en remettre à un arbitrage chariatique suscitent une vague de critiques qui s'étend jusque dans ses propres rangs. ${ }^{54}$ Deuxièmement, la politique d'intimidation menée par al-Nusra a pour effet d'encourager certaines petites factions à acheter leur sécurité en versant un tribut financier au groupe jihadiste. Empêcher d'autres unités rebelles de graviter à leur tour dans l'orbite d'al-Nusra et de renforcer encore davantage ce dernier implique donc, pour Ahrar al-Sham, de se présenter en rempart efficace contre les menées des jihadistes.

Les tensions locales al-Nusra surviennent en parallèle à l'approfondissement du rapprochement politique entre Ahrar al-Sham et les factions rebelles situées sur sa gauche. La participation à la conférence de Riyad aux côtés de ces dernières constitue assurément à ce jour le développement le plus importante dans ce processus, qui se manifeste également à travers des gestes symboliques. Dans la foulée de la conférence de Riyad, un collectif de factions rebelles non jihadistes allant des groupes soutenus par le Pentagone aux salafistes de Jaych al-Islam prend pour habitude de publier des communiqués communs arborant le drapeau national et le titre « Armée Syrienne Libre ». Jusqu'en septembre 2016, Ahrar al-

\footnotetext{
${ }^{51}$ Entretien avec Bilal Jabiro, membre d'Ahrar al-Sham et gouverneur d'Idlib à l'époque, Alep, août 2016.

52 « L'Instance Islamique demande à al-Nusra de livrer les agresseurs dans l'affaire de Kafr Nubbul ", Al-Durar al-Shamiyya, 13 juillet 2015 ; "Communiqué du Front al-Nusra concernant l'incident de Dana » (en arabe), 2 octobre 2015,

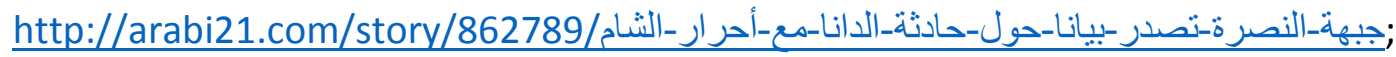
" Accrochages entre Ahrar al-Sham et le Front al-Nusra à Salqin », Enab Baladi, 24 janvier 2016, http://www.enabbaladi.net/archives/61902.

53 « Le Front al-Nusra attaque les bases de l'ASL au Nord d'Idlib » (en arabe), Enab Baladi, 21 décembre 2015, http://www.enabbaladi.net/archives/61902; " Al-Nusra arrête le chef de Jaysh al-Tahrir, Orient, 3 juillet 2016, http://orientnews.net/ar/news_show/116542/0/النصرة-تعتقل-قائد-جيش-التحرير -وتستولي-على-مقر اته-بريف-إدلب. ${ }^{54}$ Tweet du juge égyptien membre d'al-Nusra Sharif Hazza', 7 juillet 2016, https://twitter.com/Sherifhazzaa/status/750872612318932992.
} 
Sham se refuse à signer ces déclarations conjointes, préférant publier ses propres communiqués (exempts de drapeau national et de référence à l'ASL) même lorsque ces derniers reproduisent mot pour mot ceux des autres factions. ${ }^{55} \mathrm{Ce}$ souci de distinction est vertement critiqué dans les milieux islamistes anti-jihadistes, ${ }^{56}$ aux opinions desquels Ahrar al-Sham démontre sa sensibilité en décidant finalement d'apposer sa signature communiqué publié non seulement par les factions rebelles mais aussi par la Coalition Nationale pour dénoncer le déplacement forcé de la population du quartier rebelle homsiote d'al-Wa'r. ${ }^{57}$

Paradoxalement, l'accentuation des tensions locales et divergences politiques entre Ahrar alSham et le Front al-Nusra s'accompagne d'une intensification des négociations concernant leur possible fusion. L'idéal d'unité des groupes rebelles islamistes est essentiellement promu par al-Nusra dans une quête d'hégémonie, le groupe étant confiant dans l'avantage que lui confère une cohésion et des qualités organisationnelles supérieures à celle de ses partenaires. Dans cette entreprise, la branche d'al-Qaeda bénéficie du concours de hérauts de l'unité rebelle tels qu'Abdallah al-Muhaysini, un jeune religieux saoudien établi en Syrie qui, fort d'une activité frénétique en termes de propagande médiatique et de levée de fonds privés dans le Golfe, a été désigné magistrat en chef de Jaysh al-Fath et s'est imposé comme un médiateur incontournable dans les conflits entre factions. Ahrar al-Sham se montre hésitant face aux projets d'unité, par crainte des ambitions hégémoniques d'al-Nusra ainsi que des répercussions en termes de respectabilité internationale. Cependant, le groupe est prisonnier d'une contrainte normative, en l'occurrence l'idéal - littéralement incontestable - d'unité des factions rebelles islamistes.

Pour échapper au dilemme que pose les négociations avec al-Nusra, Ahrar al-Sham, toutes ailes confondues, fait de la rupture des liens entre al-Nusra et al-Qaeda une condition à la réalisation de 1'unité. ${ }^{58}$ Or, en juillet 2016, al-Nusra se rebaptise Fath al-Sham et se déclare officiellement indépendant de toute organisation étrangère. Au sein d'Ahrar al-Sham, la mue de l'ancien al-Nusra et l'entame conséquente de nouvelles négociations sur la fusion des deux organisations ravive les divergences : tandis que, fin août, le porte-parole militaire Abu Yusuf al-Muhajir, proche des durs, annonce un mariage imminent, ${ }^{59}$ le bureau politique temporise en soulignant que la rupture bienvenue des liens entre Fath al-Sham et al-Qaeda doit d'abord

\footnotetext{
${ }^{55}$ Voir le « Communiqué concernant la trêve proposée suite au récent accord russoaméricain » (en arabe),12 septembre 2016, https://twitter.com/islamic front/status/775410192469065728 (Ahrar al-Sham) et https://www.jaishalislam.org/statement/130 (ASL).

${ }^{56}$ " Des auteurs, chercheurs et activistes critiquent Ahrar al-Sham » (en arabe), Al-Durar alShamiyya, 13 septembre 2016, http://eldorar.com/node/102961.

57 " La Coalition et les factions révolutionnaires menacent de reconsidérer le processus politique » (en arabe), Al-Durar al-Shamiyya, 19 septembre 2016, http://www.all4syria.info/Archive/345953.

58 "Chef d'Ahrar al-Sham: les liens entre al-Nusra et al-Qaeda font du tort au people syrien et à sa revolution", Aksalser, 17 avril 2015, https://www.aksalser.com/news/2015/04/17/-قائد

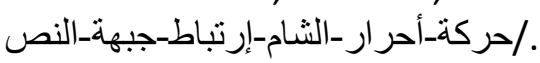

59 "Why did Jabhat Fatah al-Sham fail to merge with Syrian rebels?", Now, 20 septembre 2016, https://mobile.mmedia.me/lb/en/commentaryanalysis/567363-why-did-jabhat-fatahal-sham-fail-its-attempted-merger-with-syrianrebels.
} 
s'accompagner d'une évolution idéologique. ${ }^{60}$ Plus grave, les négociations sur la fusion contribuent au schisme le plus important dans l'histoire d'Ahrar al-Sham en encourageant Suqur al-Sham à reprendre son indépendance par crainte de voir son influence diluée dans une entité plus large. ${ }^{61}$

Ce sont les données du terrain qui auront finalement raison de ce dernier round de négociations sur l'unification d'Ahrar al-Sham et Fath al-Sham. En octobre 2016, en effet, la première faction entre en guerre ouverte contre Jund al-Aqsa, une formation jihadiste ultraradicale qui avait activement épaulé al-Nusra dans la « campagne de l'Émirat » en 2014-2015. Soupçonnée de longue date de sympathies pour l'EI, Jund al-Aqsa avait été impliqué, via certains de ses membres, dans une longue série d'assassinats perpétrés dans la province d'Idlib contre des cadres insurgés, dont des membres Ahrar al-Sham. ${ }^{62}$ Les soupçons d'allégeance secrète à l'EI avait été renforcés par le fait qu'en octobre 2015, Jund al-Aqsa avait quitté Jaysh al-Fath pour marquer son opposition à un communiqué promettant de combattre non seulement le régime mais aussi l'EI. Plusieurs incidents armés se produisent entre Jund al-Aqsa et Ahrar al-Sham en 2016, qui prennent une tournure inédite en octobre lorsque des éléments du premier groupe tentent d'enlever un commandant du second. Ahrar al-Sham répond cette fois en déployant toute sa puissance militaire en sus d'une violence rhétorique inouïe, al-Masri appelant à «en finir une fois pour toute » avec ce qu'il qualifie $d$ ' « infection pernicieuse $» .{ }^{63}$ Mis en déroute, Jund al-Aqsa ne trouve son salut qu'en se dissolvant dans Fath al-Sham, un fait accompli qu'Ahrar al-Sham accepte de mauvaise grâce et qui donne de nouveaux arguments aux opposants de l'union avec Fath al-Sham. ${ }^{64}$ À l'heure où nous écrivons ces lignes, toutefois, les désastres militaires subis par les rebelles à Alep remettent au centre la question d'unité, qui apparaît désormais comme une question existentielle pour l'insurrection.

\section{Conclusion}

Bien que le processus fût marqué de revers, l'évolution idéologique d'Ahrar al-Sham s'est soldée par une consolidation de sa posture révisionniste, et donc par un prudent rapprochement avec la sphère révolutionnaire. Deux facteurs structurels issus de la phase de genèse du groupe ont joué ici un rôle fondamental. Le premier est le partenariat noué très tôt avec des États étrangers, dont l'influence est manifeste lors d'épisodes comme la Charte d'Honneur Révolutionnaire de 2014, la conférence de Riyad l'année suivante, et l'intervention militaire turque en 2016. Le second facteur est le caractère institutionnalisé et collégial de la direction d'Ahrar al-Sham, qui non seulement empêche les faucons d'établir une hégémonie durable en dépit des mutations opérés durant le mandat de Hachim al-Cheikh, mais joue tendanciellement en faveur de leurs adversaires dans la mesure où l'expansion du

\footnotetext{
60 « [lyyad al-Sha'ar] : nous saluons la rupture même si elle est tardive ; l'évolution des idées prends du temps ", Al-Durar al-Shamiyya, 22 août 2016.

61 "Suqur al-Sham quitte Ahrar al-Sham », Enab Baladi, 3 septembre 2016, http://www.enabbaladi.net/archives/101376.

${ }^{62}$ Abazeid, " Ahrar al-Sham après une longue année », pp. 33-35.

63 " Déclaration du commandant en chef d'Ahrar al-Sham Muhannad al-Masri concernant les affrontements avec Jund al-Aqsa ", Youtube, 12 octobre 2016, https://www.youtube.com/watch?v=-lug3PjoAbU.

${ }^{64}$ "Le porte-parole de Fath al-Sham répond au chef d'Ahrar al-Sham : nous avons accepté l'allégeance de combattants valeureux » (en arabe), Al-Durar al-Shamiyya, 13 octobre 2016, http://eldorar.com/node/104159.
} 
mouvement par absorption de groupes non-jihadistes conduit à l'intégration de nouveaux leaders de sensibilité identique au sein du Conseil Consultatif.

Si le rôle des autres groupes jihadistes et en particulier d'al-Nusra/Fath al-Sham est avant tout de freiner l'évolution pragmatique d'Ahrar al-Sham en exerçant une pression discursive sur l'aile dure du mouvement et, partant, en menaçant la cohésion de ce dernier, les effets secondaires induits par l'action de ces mêmes jihadistes ont, eux, joué en sens inverse. Ainsi, les mouvements de réaction anti-jihadiste provoqués par le comportement de l'EI, en 2014, et celui d'al-Nusra, en 2015, font office d'accélérateur de la transformation idéologique d'Ahrar al-Sham en démontrant la résilience de l'opinion révolutionnaire et, partant, la nécessité persistante de rencontrer ses attentes minimales. À leur tour, les critiques formulées par les jihadistes contre ces nouvelles étapes dans l'évolution du groupe contraignent ses leaders à une justification théorique de leur posture, modifiant de ce fait la structure normative du débat. Enfin, la politique agressive menée sur le terrain par les jihadistes afin d'étendre leur influence aux dépens des factions ASL mais aussi, dans une moindre mesure, d'Ahrar alSham, ne rencontre d'abord qu'une timide réaction, du fait des impératifs de la coopération militaire, avant de rencontrer, du fait de l'accentuation de la menace, une résistance plus ferme illustrée en particulier par l'écrasement de Jund al-Aqsa.

Bien que consolidé, le révisionnisme d'Ahrar al-Sham n'est pas irréversible. Alors que l'intervention irano-russe réalise ses objectifs, avec la reprise d'Alep-Est par les loyalistes et l'extinction progressive de la présence rebelle dans les banlieues de Damas, les bénéfices du pragmatisme risquent fort d'être questionnés et des options comme la fusion avec Fath alSham, reconsidérées. La re-radicalisation d'Ahrar al-Sham demeure donc tout à fait possible mais, si elle venait à se produire, on espère avoir fourni dans ces pages les clés permettant d'y voir le fruit d'un processus contingent plutôt que d'un quelconque atavisme. 